Témoigner Témoigner. Entre histoire et mémoire

Getuigen Revue pluridisciplinaire de la Fondation Auschwitz

$121 \mid 2015$

Violences radicales en scène

\title{
Rwanda : Épisode 5 - Rivières et marais rwandais : lieux de mémoire du génocide?
}

Rwanda aflevering 5. Rwandese rivieren en moerassen: herinneringsplekken van de volkerenmoord?

\section{Rémi Korman}

\section{(2) OpenEdition}

Journals

Édition électronique

URL : https://journals.openedition.org/temoigner/3597

DOI : 10.4000/temoigner.3597

ISSN : 2506-6390

Traduction(s) :

Rwanda aflevering 5. Rwandese rivieren en moerassen: herinneringsplekken van de volkerenmoord? URL : https://journals.openedition.org/temoigner/3602 [nl]

Éditeur :

Éditions du Centre d'études et de documentation Mémoire d'Auschwitz, Éditions Kimé

Édition imprimée

Date de publication : 1 octobre 2015

Pagination : 182-185

ISSN : 2031-4183

Référence électronique

Rémi Korman, «Rwanda : Épisode 5 - Rivières et marais rwandais : lieux de mémoire du génocide? », Témoigner. Entre histoire et mémoire [En ligne], 121 | 2015, mis en ligne le 01 octobre 2016, consulté le 04 février 2022. URL : http://journals.openedition.org/temoigner/3597 ; DOI : https://doi.org/10.4000/ temoigner.3597 


\section{Rwanda \\ . \\ - \\ RIVIĖRES ET MARAIS RWANDAIS : LIEUX DE MEMOIRE DU GENOCIDE ?}

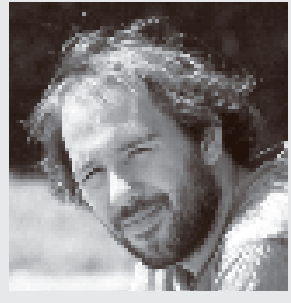

$\diamond$ Par Rémi Korman, EHESS (École des Hautes Etudes en Sciences Sociales). II effectue des recherches universitaires sur la mémoire du génocide des Tutsi au Rwanda où il se rend régulièrement. Il développe en parallèle des projets de transmission publique de cette mémoire avec l'association lbuka à Lyon.

$\diamond$ Cette chronique régulière vise à présenter la façon dont se construit le souvenir du génocide des Tutsi au Rwanda depuis 1994. Elle prendra en compte les spécificités du modèle mémoriel rwandais, mais aussi les nombreux liens tissés avec des institutions travaillant sur la mémoire de la Shoah, sur le génocide des Arméniens ou encore le génocide cambodgien.

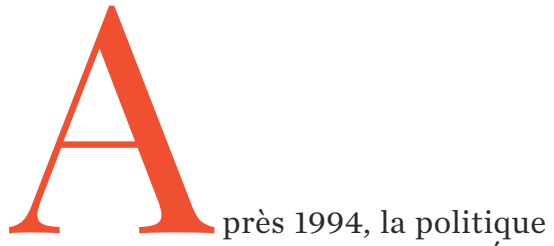
de mémoire mise en place par l'État rwandais est centrée sur la question des corps des victimes. Leur exhumation et inhumation en dignité est alors au cœur du processus commémoratif. Mais pour les rescapés, comment organiser une cérémonie du souvenir en l'absence de corps ? Est-il possible de commémorer de façon plus symbolique? Que signifie célébrer la mémoire lorsqu'il n’y a ni corps et ni site mémorial consacré?

C'est autour de ces questions qu'apparaissent depuis le milieu des années 2000 de nouvelles formes commémoratives du génocide. En dehors des cérémonies mises en place par l'État, des associations de survivants essayent de se souvenir autrement des victimes du génocide contre les Tutsi, en organisant des commémorations thématiques. On peut citer le cas du Groupement des anciens de l'association des étudiants rescapés du génocide, plus connu sous le nom de GAERG, qui organise chaque année une commémoration pour les familles entièrement disparues (Imiryango yazimye muri jenoside yakorewe abatutsi). Mais aussi, et ce sera l'objet de cet article, le cas des commémorations organisées par de nombreuses associations de rescapés, comme Dukundane Family, en mémoire des Tutsi jetés dans les cours d'eau, les lacs et les marais

\section{LA RIVIÈRE NYABARONGO}

\section{OU " PAR LE RACCOURCI "}

Revenir sur le rôle des rivières au cours du génocide, c'est revenir sur l'histoire des représentations de la population rwandaise produites à l'époque coloniale. Le Rwanda, décrit dès la fin du XIX ${ }^{\mathrm{e}}$ siècle comme le pays des Sources du Nil dont la Nyabarongo serait l'un des principaux affluents, est 


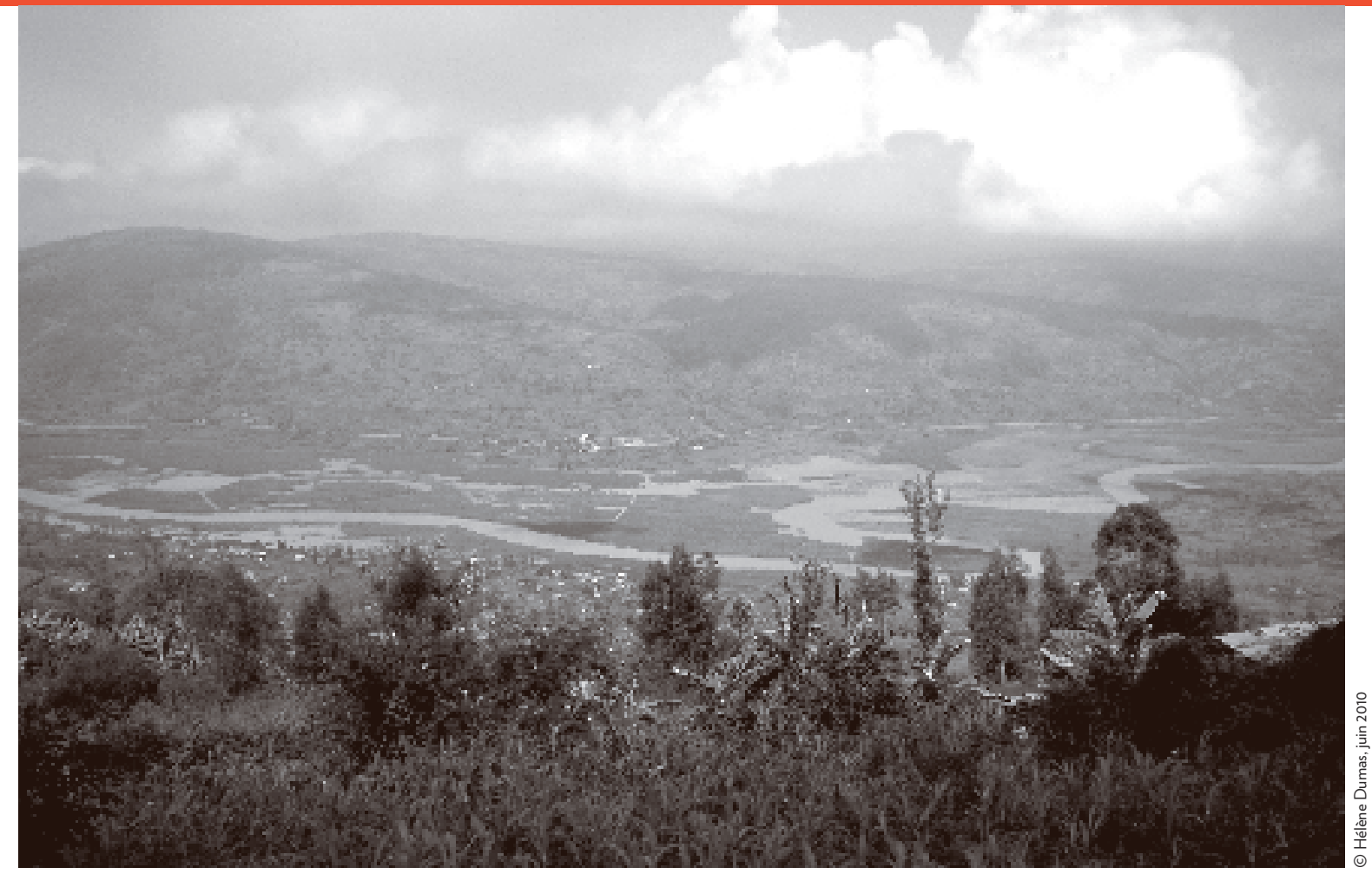

considéré par les colonisateurs comme un royaume particulier. L'aristocratie tutsie impressionne fortement les premiers Européens, qui élaborent ensuite diverses théories sur le peuplement et les migrations au Rwanda. Largement fantasmées et le plus souvent délirantes, ces théories présentent la population tutsie comme une "race » extérieure au Rwanda. L’idéologie développée, que le chercheur Jean-Pierre Chrétien nomme l'idéologie hamitique, consiste à voir dans les Tutsi un peuple originaire d'Éthiopie ou d'Abyssinie (Chrétien ; Kabanda, 2013). Cette idéologie, qui était en partie positive, les Tutsi étant classifiés comme des "faux-nègres », voit son sens renversé aux abords de l'indépendance. Ce qui était vu alors comme une qualité est devenue un stigmate. Les Tutsi sont alors décrits comme des envahisseurs, et plus tard, comme des parasites.
Dès les premiers massacres en 1959, des Tutsi sont jetés dans les cours d'eau au Rwanda, en particulier dans les affluents de la Nyabarongo. Mais c'est véritablement au début de la guerre civile que la Nyabarongo devient dans les discours politiques un lieu de l'expression génocidaire. À l'occasion d'un meeting organisé à Kabaya en 1992, l'intellectuel rwandais Léon Mugesera prononce un discours désormais célèbre, appelant à renvoyer les Tutsi en Éthiopie, «par le raccourci ». Il dit ainsi : « Ne sais-tu pas donc ni écouter ni lire? Moi, je te fais savoir que chez toi, c'est en Éthiopie, que nous vous ferons passer par la Nyabarongo pour que vous parveniez vite là-bas. »

De fait, au cours du génocide, plusieurs dizaines de milliers de Tutsi sont jetés dans les cours d'eau, morts ou vivants. Les cadavres arriveront par milliers dans le lac Victoria en Ouganda, • •
_ La rivière Nyabarongo depuis la colline de Nyarusange à Shyorongi. 
- à plus de $1000 \mathrm{~km}$ de distance. Le destin singulier de ces victimes a fait l'objet d'un documentaire par l'artiste rwandais, lui-même rescapé du génocide, Dady de Maximo. Intitulé «Par le raccourci », en référence à l'expression utilisée au cours du génocide, ce film est peu diffusé en dehors du Rwanda, en raison de la répétition lancinante de scènes d'une grande violence. Dady de Maximo revient longuement sur l'histoire de la Nyabarongo, de ses représentations, et des massacres qui s'y sont déroulés. Surtout, il raconte l'histoire de ces dizaines de milliers de corps repêchés du lac Victoria, et enterrés par des pêcheurs ougandais.

Si plusieurs sites mémoriaux existent en Ouganda depuis 1994, l'État rwandais souhaite construire trois grands monuments à Ggolo, Kanseselo et Lambu. Déjà, la diaspora rwandaise

_Affiche du documentaire de Daddy de Maximo, Par le Raccourci, 2009.

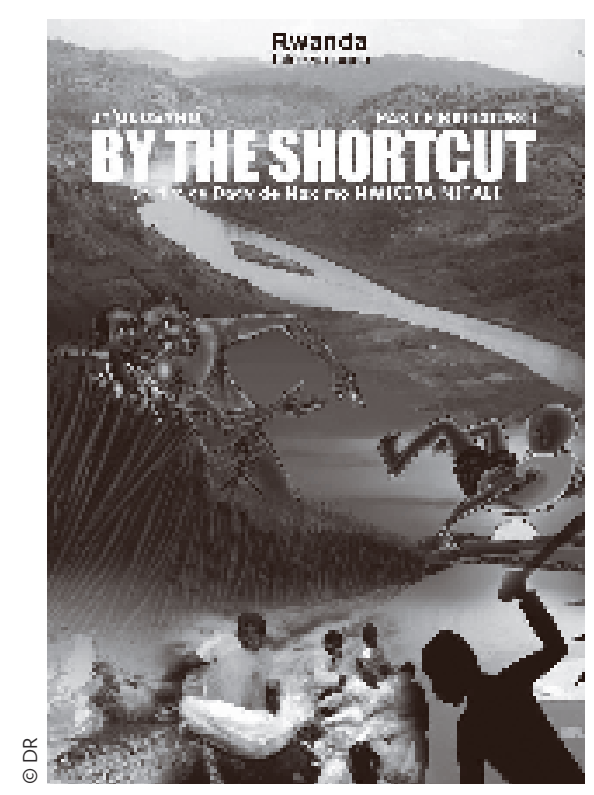

en Ouganda y organise chaque année des commémorations. Des pèlerinages sont aussi préparés depuis le Rwanda. C'est dans ce contexte que la Nyabarongo est devenue, selon l'historien Philibert Gakwenzire, " un lieu de mémoire » (Gakwenzire, 2010).

\section{IBISHANGA OU LES MARAIS RWANDAIS}

Rivières et autres cours d'eau ne sont pas les seuls sites naturels transformés en lieux de massacre. Le rôle des marais dans le génocide commis dans la commune de Nyamata située dans le Bugesera a d'ailleurs fait l'objet de nombreuses recherches et d'une intense production littéraire.

Scholastique Mukasonga raconte dans Inyenzi ou les cafards, l'histoire de la déportation de nombreux Tutsi en 1959, à l'époque coloniale, dans cette région marécageuse (Mukasonga, 2006). Ce déracinement forcé, qui n'a pas encore fait l'objet d'étude historique, explique la forte concentration de population tutsie dans la commune de Nyamata au moment du génocide.

Jean Hatzfeld y revient dans son premier ouvrage publié en 2000, Dans le nu de la vie : récits des marais rwandais. Dans tous les récits des rescapés qu'il interroge, les marais reviennent aussi comme une frontière indépassable de la commune, bordée par les rivières Akanyaru, Mwogo et Nyabarongo. Pour les rescapés, les marais sont un lieu ambivalent. Il s'agit en 1994 d'un lieu de cachette et de survie, il est possible d'y apaiser sa soif grâce à l'eau contenue dans la canne à sucre. Mais c'est aussi et surtout un lieu de mort, avec l'eau stagnante, le risque de la malaria, et les tueurs qui pourchassent et attaquent tous les jours. 
Une rescapée interrogée, Berthe Mwanankabandi raconte ainsi :

\begin{abstract}
Nous avons couru sans réflexion pour atteindre les marais de Nyamwiza. Le soir, la pluie se déversait sans jamais s'interrompre et nous avons cherché refuge à l'école de Cyugaro, dans le bois d'eucalyptus, non loin des marais. Cela devait délimiter notre programme de marche pendant un mois : les marais, l'école, les marais. [...] Tous les matins, je préparais aux enfants de la nourriture avec des aliments arrachés des parcelles; ensuite je les emmenais à l'avance se dissimuler sous les feuillages des papyrus en compagnie des grandes personnes qui avaient épuisé leur énergie. Il fallait, les jours de soleil, changer d'endroit, à cause des empreintes de pied laissées dans la boue séchée. Quand les massacreurs arrivaient, ils chantaient ; et c'était notre tour d'aller nous éparpiller dans les marais. (Hatzfeld, 2000, p. 176)
\end{abstract}

Combien sont morts dans les marais rwandais à Nyamata, et ailleurs? Quelle sépulture symbolique pour ces victimes dont les corps ne peuvent être exhumés des marais ? Là encore, de nouvelles formes commémoratives ont émergé ces dernières années.

En avril 2015, des stèles ont ainsi été érigées «à la lisière du marais mouvant de Ntarama qui a englouti plus de 2000 Tutsis pendant le génocide de 1994. [...] Également appelé "marais de Rwimpiri (ou le repère des vipères)”, ce marais, morne au pied d'un bois d'eucalyptus sur le sol rocailleux d'une colline abrupte, fut effectivement le repère de la mort en avril 1994.» (Rugiririza, 2015)

Ces commémorations jusque-là inconnues montrent la prise en compte par les associations de rescapés, mais aussi par l'État rwandais, de tous les types de sites concernés par le génocide. Elle va à l'encontre de la politique de mémoire centralisée menée depuis 1994 qui a surtout consisté en la construction d'importants mémoriaux nationaux sur les sites de massacre, ayant chacun une symbolique propre. Le mémorial de Nyanza de Kicukiro symbolise l'inaction de l'ONU en 1994, le site de Bisesero la résistance des populations tutsies. Les sites de Nyamata, Ntarama ou encore Nyarubuye rappellent les massacres dans les églises catholiques. Le mémorial de Murambi dénonce lui le rôle de la France, le site ayant été le siège de l'opération Turquoise.

Face à cette production de symbolique nationale et diplomatique, les nouvelles formes commémoratives rendent compte d'une décentralisation des pratiques mémorielles et d'une volonté par les associations de rescapés de revenir sur les multiples formes d'exécution du génocide au niveau local.

\section{BIBLIOGRAPHIE}

- Chrétien, Jean-Pierre ; Kabanda, Marcel (2013):

Rwanda, racisme et génocide : l'idéologie hamitique, Paris, Belin.

- De Maximo, Dady (2009) : Par le raccourci, Kigali, Dadmax.

- Gakwenzire, Philibert (mars 2010) : "Nyabarongo, une rivière du supplice », in Dialogue $\mathrm{n}^{\circ} 190$, p. 823.

- Hatzfeld, Jean (2000) : Dans le nu de la vie. Récits des marais rwandais, Paris, Le Seuil.

- Rugiririza, Ephrem (21/04/2015) : « Un marais mouvant érigé en mémorial du génocide », in Agence Hirondelle.

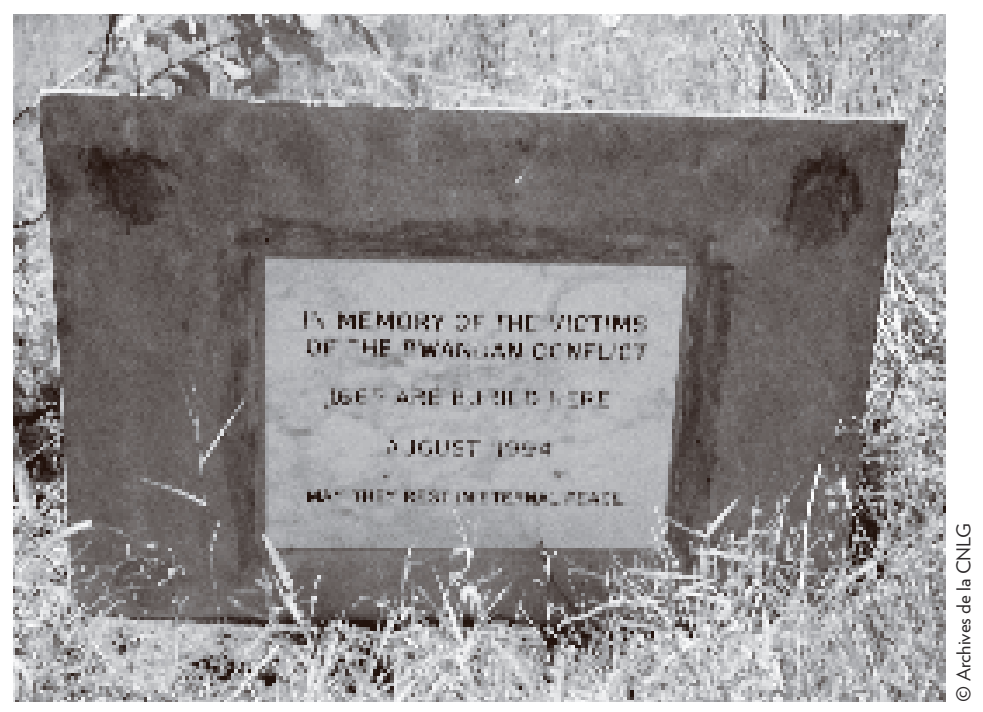

